\title{
Hiperhidrosis axilar primaria
}

Primary axillary hyperhidrosis

\section{Evelyne Halpert' ${ }^{1}$, Clara Inés Ortiz², Daniela Chaparro³}

1. Médica dermatóloga, especialista en Dermatología Infantil y en Epidemiología Clínica; jefe, Sección de Dermatología Pediátrica, Fundación Santa Fe de Bogotá, Bogotá, D.C., Colombia

2. Médica dermatóloga, Fundación Santa Fe de Bogotá, Bogotá, D.C., Colombia

3. Médica, especialista en Epidemiología Clínica; asistente de investigación, Asociación Médica de los Andes, Bogotá, D.C., Colombia

\section{RESUMEN}

Introducción. La hiperhidrosis axilar primaria representa un motivo de consulta muy frecuente en la práctica dermatológica. Las alternativas de tratamiento no quirúrgico son de alto costo y, los resultados, de corta duración. Dados el impacto y la cronicidad de esta condición, los pacientes solicitan tratamientos no invasivos y duraderos para el manejo de la hiperhidrosis. Nos enfrentamos a mucha publicidad en cuanto a nuevas opciones terapéuticas, tales como láser, radiofrecuencia y microondas. Esta revisión pretende contribuir a responder la pregunta: ¿es posible ofrecer a los pacientes tratamientos como láser, radiofrecuencia y microondas para la hiperhidrosis axilar primaria?

Metodología. Se hizo una revisión sistemática de la literatura científica utilizando las bases de datos Pubmed, Cochrane y Lilacs, teniendo como población diana a los pacientes mayores de 18 años con diagnóstico de hiperhidrosis axilar primaria.

Resultados. Se encontraron 162 artículos y, a partir de ellos, se elaboró la siguiente revisión de tema.

Conclusiones. La termoterapia (láser, microondas, radiofrecuencia pixelada) se ha utilizado en los últimos años con resultados muy variables y, aunque es claro su efecto en cuanto a la destrucción de las glándulas, aún no se dispone de suficiente información de la duración del efecto, la temporalidad de las sesiones, y la necesidad y frecuencia de mantenerlas. Se necesita hacer más estudios en nuestra población.

PALABRAS CLAVE: hiperhidrosis axilar, tratamiento, termoterapia

\section{SUMMARY}

Introduction: Primary axillary hyperhidrosis represents a frequent reason for consultation in dermatological practice. Non-surgical treatment alternatives are costly and short-term results. Given the impact and chronicity of this condition, patients request non-invasive and long-lasting treatments for the management of hyperhidrosis. We are facing a lot of publicity regarding new therapeutic options such as laser, radiofrequency and microwave.
Correspondencia:

Daniela Chaparro

\section{Email:}

ld_chr@hotmail.com

Recibido: 20/02/2017

Aceptado: 29/06/2017

Conflictos de interés:

No se reportan conflictos de interés.

Financiación:

Ninguna. 
This review aims to answer the question if it is possible to offer patients treatments such as laser, radiofrequency and microwaves for primary axillary hyperhidrosis.

Methodology: We did a systematic review of the literature using the Pubmed, Cochrane and Lilacs databases, having as target population patients older than 18 years of age with diagnosis of primary axillary hyperhidrosis.

Results: A total of 162 articles were found and from these, the following revision was prepared.

Conclusions: Thermotherapy (laser, microwave, pixelated radiofrequency) has been used in the last years with very variable results and although its effect on the destruction of the glands is clear, there is not enough evidence on the duration of the effect, the temporality of the sessions and the need and frequency of maintenance. We need to carry out studies in our population.

KEY WORDS: axillary hyperhidrosis, treatment, thermotherapy

\section{"Esta condición representa un motivo de consulta frecuente en la práctica dermatológica. Las alternativas de tratamiento no qui- rúrgico son de alto costo y los resultados son de corta duración. Dados el impacto y la cronicidad de esta condición, los pacientes solicitan tratamientos no invasivos y duraderos para el manejo de la hiperhidrosis."}

\section{INTRODUCCIÓN}

La habilidad para percibir y regular la temperatura corporal es una característica fundamental para la supervivencia humana ${ }^{1}$. Los mecanismos fisiológicos para lograr el equilibrio en la temperatura corporal son, principalmente, la termogénesis y la sudoración.

La hiperhidrosis es definida como la sudoración excesiva, más allá de lo esperado para las condiciones ambientales y fisiológicas ${ }^{2}$. Es una condición crónica que afecta en forma significativa el desempeño social y laboral, y por lo tanto, la calidad de vida de los pacientes que la padecen.

Esta condición representa un motivo de consulta frecuente en la práctica dermatológica. Las alternativas de tratamiento no quirúrgico son de alto costo y los resultados son de corta duración. Dados el impacto y la cronicidad de esta condición, los pacientes solicitan tratamientos no invasivos y duraderos para el manejo de la hiperhidrosis. Actualmente, nos enfrentamos a nuevas opciones terapéuticas, tales como láser, radiofrecuencia y microondas.

Esta revisión pretende contribuir a responder la pregunta: ¿es posible ofrecer a los pacientes tratamientos como láser, radiofrecuencia y microondas para la hiperhidrosis axilar primaria?

\section{METODOLOGÍA}

Se llevó a cabo una revisión sistemática de la literatura utilizando las bases de datos Pubmed, Cochrane y Lilacs, teniendo como población diana a los pacientes mayores de 18 años con diagnóstico de hiperhidrosis axilar primaria. Se incluyeron en la búsqueda los términos MESH: 'hyperhidrosis', 'primary axillary treatment', 'therapy', 'microwave', 'laser’ y 'radiofrequency'.

Se filtraron los resultados por fecha de publicación; se incluyeron únicamente estudios en inglés oespañol, publicados entre $1^{\circ}$ de enero de 1970 y el 31 de diciembre del 2016. Los tipos de estudios incluidos fueron: revisiones de tema, revisiones sistemáticas, ensayos clínicos, estudios observacionales y reportes de caso. Se encontraron 162 artículos y, a partir de ellos, se elaboró la siguiente revisión. 


\section{MECANISMO DE LA SUDORACIÓN}

La sudoración es un proceso fisiológico vital para el ser humano. Existen dos tipos de sudoración, la termorreguladora y la emocional. Además del ser humano, pocos animales tienen la habilidad de evaporar el calor mediante la activación de las glándulas sudoríparas ecrinas ${ }^{3}$.

La hiperhidrosis es la producción de sudor en cantidades superiores a las fisiológicamente requeridas para la termorregulación corporal. A pesar de que la hiperhidrosis no es una condición fatal, altera en forma significativa la calidad de vida del paciente, debido a su impacto psicológico y social ${ }^{4}$. Afecta entre el 1 y el $3 \%$ de la población general, y no se encuentra diferencia entre sexos. En el 2004, Strutton, et al., encontraron una prevalencia de hiperhidrosis del 2,8 \% en los Estados Unidos y, de hiperhidrosis axilar, en el 50,8 $\%$ de los casos ${ }^{5}$. No hay datos de prevalencia de este trastorno en la población colombiana.

El incremento de la temperatura corporal, ya sea por termogénesis o por irradiación solar, estimula los receptores de calor y frío situados en la piel y en las vísceras ${ }^{6}$. Los impulsos son enviados al sistema nervioso central a lo largo de las fibras A y C. Las neuronas termosensitivas centrales están situadas en la médula espinal, el tronco cerebral y el hipotálamo. Este último es el centro de integración de todas las aferencias termosensoriales y su función es mantener la temperatura corporal a 37 grados Celsius ${ }^{7}$. Esta regulación se logra por medio de termogénesis (contracciones musculares y vasoconstricción cutánea) o por liberación de calor (sudoración y vasodilatación) 8 .

El ser humano tiene cerca de 3 millones de glándulas sudoríparas. Las glándulas ecrinas están distribuidas en la superficie corporal, con altas concentraciones en las palmas, las plantas y la frente ${ }^{3}$. La inervación de las glándulas sudoríparas es simpática y posgan- glionar. El principal neurotransmisor involucrado es la acetilcolina ${ }^{9}$.

Las glándulas ecrinas son tubulares, con sus porciones secretoras enrolladas, y habitualmente están localizadas en las zonas profundas de la dermis o, con mayor frecuencia, en la hipodermis. El conducto sudoríparo asciende a través de la dermis y la epidermis para abrirse en un poro sudoríparo en la superficie cutánea. Cada una de las glándulas pesa de 30 a 40 \%g. Las personas que hacen ejercicio intenso en un ambiente cálido, pueden sudar hasta 10 litros por día. Estas glándulas son funcionales desde el nacimiento, no tienen conexión con los folículos pilosos y su producto es exclusivamente acuoso. La porción secretora de la glándula está revestida por un epitelio cúbico o cilíndrico bajo que contiene dos tipos de células secretoras: las células oscuras y las claras.

El segundo tipo de glándulas sudoríparas, las glándulas apocrinas, se encuentra en las axilas, el monte de Venus y la región perianal. Estas glándulas son de mayor tamaño que las ecrinas. Su porción secretora enrollada puede ser de $3 \mathrm{~mm}$ de diámetro, en comparación con los 0,4 mm de las glándulas ecrinas. Están localizadas en la dermis y sus conductos se abren en el canal de un folículo piloso. Su actividad secretora se inicia en la pubertad. El producto de las glándulas apocrinas es un líquido ligeramente viscoso que es inodoro pero, cuando es modificado por las bacterias residentes en la piel, adquiere un olor desagradable que ocasiona dificultades sociales.

En las mujeres, las glándulas apocrinas de las axilas presentan cambios citológicos periódicos durante el ciclo menstrual. En el período premenstrual, hay hipertrofia de las células y de la luz, seguida de una regresión durante la menstruación ${ }^{10}$.

En la tabla 1 se resumen las principales diferencias entre las glándulas ecrinas y las apocrinas.

CARACTERÍSTICAS DE LAS GLÁNDULAS SUDORÍPARAS

Glándulas ecrinas

Sin conexión con los folículos pilosos

Funcionales desde el nacimiento

Secreción acuosa

Inervación colinérgica

\section{Glándulas apocrinas}

Apéndices de los folículos pilosos

Funcionales en la pubertad

Secreción lipídica

Inervación adrenérgica

Tabla 1. Características de las glándulas sudoríparas 


\begin{abstract}
1. Mi sudoración nunca se nota y nunca interfiere con mis actividades diarias.
2. Mi sudoración es tolerable pero a veces interfiere con mis actividades diarias.

3. Mi sudoración es poco tolerable y frecuentemente interfiere con mis actividades diarias.

4. Mi sudoración es intolerable y siempre interfiere con mis actividades diarias.
\end{abstract}

Tabla 2. Escala de seriedad de la hiperhidrosis

\section{CLASIFICACIÓN}

La hiperhidrosis se puede clasificar según su etiología (primaria o secundaria) ${ }^{11}$ y según su localización (focal o generalizada) ${ }^{12}$.

- Hiperhidrosis primaria: generalmente, se presenta en edad temprana, la infancia o la adolescencia. La hiperhidrosis primaria ocurre como consecuencia de una disfunción neuronal autonómica. Se presenta en las áreas de mayor concentración de glándulas ecrinas, como son las palmas, las plantas y las axilas 13. Otras localizaciones menos frecuentes incluyen el cuero cabelludo, el tronco y la cara ${ }^{14}$. Su diagnóstico requiere que se hayan descartado otras causas, como las de la hiperhidrosis secundaria.

- Hiperhidrosis secundaria: esta sudoración excesiva se debe a enfermedades sistémicas, como diabetes mellitus, hipertiroidismo, hiperparapituitarismo, trastornos neurológicos, malformación de ArnoldChiari, feocromocitoma, enfermedades respiratorias y trastornos psiquiátricos. Generalmente, la sintomatología empieza en la vida adulta. La hiperhidrosis asimétrica sugiere enfermedad neurológica $^{15}$. La hiperhidrosis palmo-plantar se puede observar en pacientes con historia de alcoholismo crónico $^{16}$. Se deben solicitar los exámenes de laboratorio orientados por el interrogatorio e historia clínica de cada paciente.

\section{DIAGNÓSTICO}

Historia clínica y examen físico: la principal herramienta diagnóstica para la hiperhidrosis es la historia clínica. La percepción de sudoración por el paciente determina la seriedad de la enfermedad y, por lo tanto, la reacción terapéutica.

Los criterios diagnósticos de hiperhidrosis primaria incluyen: sudoración excesiva con duración de, al menos, 6 meses, sin una causa obvia, y debe tener, al menos, dos de las siguientes características ${ }^{17}$ :

- $\quad$ altera las actividades diarias;

- la sudoración es bilateral, relativamente simétrica y con ocurrencia de, al menos, una vez por semana;

- $\quad$ edad de inicio antes de los 25 años;

- la sudoración cesa durante el sueño, y

- hay historia familiar de sudoración excesiva.

En el examen físico de estos pacientes, se evidencia sudoración excesiva sin un factor desencadenante claro. Es importante determinar las áreas del cuerpo que están comprometidas y si la sintomatología es unilateral o bilateral.

\section{Evaluación objetiva}

Test de yodo: consiste en la aplicación de povidona yodada al $2 \%$ sobre la piel de las axilas. Se deja secar y luego se espolvorea almidón sobre la misma área. Al sudar, en presencia del yodo, el almidón toma un tono azul oscuro o morado, y así es posible visualizar y delimitar la zona afectada.

Gravimetría: consiste en comparar un papel de filtro antes y después de su aplicación en la zona explorada. El sujeto debe encontrarse en un ambiente de baja humedad y la zona en cuestión debe estar completamente seca.

\section{Escalas de evaluación}

Para el diagnóstico y la evaluación objetiva de la hiperhidrosis, es posible aplicar escalas que evalúan su seriedad e impacto en la calidad de vida de los pacientes.

La escala de seriedad de la hiperhidrosis es una herramienta específica, rápida y fácil de utilizar, que permite medir cualitativamente la seriedad de la sudoración de los pacientes ${ }^{3}$ (tabla 2 ).

En la tabla 3 se presenta el índice de la calidad de vida en dermatología (Dermatology Life Quality Index, DLQI) ${ }^{18}$, una escala que fue adaptada a los países de habla hispana, para evaluar el impacto de las enfermedades dermatológicas en la calidad de vida y en el desempeño diario de los pacientes.

En el caso de la hiperhidrosis axilar, múltiples estudios han evaluado la calidad de vida de los pacientes para medir el impacto de diferentes opciones terapéuticas $^{19,20}$. 


\begin{tabular}{|c|c|c|}
\hline 1 & $\begin{array}{l}\text { En los últimos siete días, ¿ha sentido prurito, inflamación, dolor o } \\
\text { escozor en su piel? }\end{array}$ & $\begin{array}{l}\text { Muchísimo } \\
\text { Mucho } \\
\text { Poco } \\
\text { Nada }\end{array}$ \\
\hline 2 & $\begin{array}{l}\text { En los últimos siete días, ¿se ha sentido incómodo o cohibido por } \\
\text { causa de sus problemas de piel? }\end{array}$ & $\begin{array}{l}\text { Muchísimo } \\
\text { Mucho } \\
\text { Poco } \\
\text { Nada }\end{array}$ \\
\hline 3 & $\begin{array}{l}\text { En los últimos siete días, ¿sus problemas de piel le han interferido } \\
\text { para ir de compras o en las actividades del hogar? }\end{array}$ & $\begin{array}{l}\text { Muchísimo } \\
\text { Mucho } \\
\text { Poco } \\
\text { Nada }\end{array}$ \\
\hline 4 & $\begin{array}{l}\text { En los últimos siete días, ¿sus problemas de piel han influido en la } \\
\text { ropa que llevaba? }\end{array}$ & $\begin{array}{l}\text { Muchísimo } \\
\text { Mucho } \\
\text { Poco } \\
\text { Nada }\end{array}$ \\
\hline 5 & $\begin{array}{l}\text { En los últimos siete días, ¿sus problemas de piel han afectado } \\
\text { alguna actividad social o de ocio? }\end{array}$ & $\begin{array}{l}\text { Muchísimo } \\
\text { Mucho } \\
\text { Poco } \\
\text { Nada }\end{array}$ \\
\hline 6 & $\begin{array}{l}\text { En los últimos siete días, ¿le ha sido difícil practicar un deporte a } \\
\text { causa de sus problemas de piel? }\end{array}$ & $\begin{array}{l}\text { Muchísimo } \\
\text { Mucho } \\
\text { Poco } \\
\text { Nada }\end{array}$ \\
\hline 7 & $\begin{array}{l}\text { En los últimos siete días, ¿sus problemas de piel le han impedido } \\
\text { trabajar o estudiar? Si la respuesta es «no», en los últimos siete } \\
\text { días, ¿su piel le ha causado algún problema en el trabajo o en los } \\
\text { estudios? }\end{array}$ & $\begin{array}{l}\text { Muchísimo } \\
\text { Mucho } \\
\text { Poco } \\
\text { Nada }\end{array}$ \\
\hline 8 & $\begin{array}{l}\text { En los últimos siete días, ¿su piel le ha causado problemas con su } \\
\text { pareja, algún amigo íntimo o pariente? }\end{array}$ & $\begin{array}{l}\text { Muchísimo } \\
\text { Mucho } \\
\text { Poco } \\
\text { Nada }\end{array}$ \\
\hline 9 & $\begin{array}{l}\text { En los últimos siete días, ¿sus problemas de piel le han dificultado } \\
\text { su vida sexual? }\end{array}$ & $\begin{array}{l}\text { Muchísimo } \\
\text { Mucho } \\
\text { Poco } \\
\text { Nada }\end{array}$ \\
\hline 10 & $\begin{array}{l}\text { En los últimos siete días, ¿el tratamiento de su piel ha sido un } \\
\text { problema para usted, por ejemplo, porque ha ensuciado la casa o le } \\
\text { ha quitado tiempo? }\end{array}$ & $\begin{array}{l}\text { Muchísimo } \\
\text { Mucho } \\
\text { Poco } \\
\text { Nada }\end{array}$ \\
\hline
\end{tabular}

Tabla 3. Índice de calidad de vida en dermatología (DLQI) ${ }^{21}$

\section{TRATAMIENTO}

En la actualidad, se cuenta con diferentes opciones de tratamiento ${ }^{3}$. Sin embargo, sólo la alternativa quirúrgica ofrece soluciones más permanentes.

\section{Tratamiento local}

Tópico: los medicamentos tópicos son fáciles de aplicar y de relativo bajo costo. Se considera la primera línea de tratamiento para la hiperhidrosis axilar primaria. El cloruro de aluminio es el agente más frecuentemente utilizado. Se recomienda su uso al 10-15 $\%{ }^{22}$. Su mecanismo de acción se atribuye a la interacción entre el cloruro de aluminio y la queratina de los conductos sudoríparos ${ }^{23}$. Su principal efecto secundario es la irritación local en algunos pacientes. Únicamente es 
efectivo en casos leves.

Recientemente, se ha empezado a usar el glicopirrolato (agente anticolinérgico) a concentraciones del $1 \%$ o el $2 \%$ en atomizaciones. Un estudio inicial descriptivo reciente informó su buen perfil de seguridad y eficacia al comparar su presentación al $2 \%$ con toxina botulínica, con seguimiento hasta por seis semanas ${ }^{24}$.

Iontoforesis: consiste en introducir una sustancia ionizada en la zona que se va a tratar, mediante corriente eléctrica y un medio acuoso, lo cual facilita el movimiento transdérmico de iones solubles y genera un gradiente de potencial eléctrico. El tratamiento se debe hacer diariamente durante dos semanas, con sesiones de 30 minutos, aproximadamente, intensificando gradualmente la energía hasta llegar al rango terapéutico de 10 a $18 \mathrm{~mA}^{25}$. No hay estudios clínicos aleatorizados que hayan evaluado este tratamiento. Sin embargo, en los estudios observacionales se reporta una efectividad del 80 al $100 \%$, específicamente en hiperhidrosis palmar y plantar ${ }^{26,27}$. Los efectos secundarios, que incluyen dolor, irritación, eritema y descamación, hacen que su uso en la práctica clínica no sea frecuente. La iontoforesis es la segunda línea de tratamiento en la hiperhidrosis palmar y plantar, pero para la hiperhidrosis axilar primaria, que es el foco de esta revisión, no hay estudios que sustenten su uso clínico.

Toxina botulínica: la toxina botulínica tipo A, bloquea la liberación de acetilcolina, entre otros neurotransmisores, en las membranas presinápticas ${ }^{28} \mathrm{y}$ en las glándulas ecrinas, mediante la activación de las proteínas SNARE. Su uso para el tratamiento de la hiperhidrosis axilar primaria fue aprobado en los Estados Unidos desde el 2004 como inyecciones transdérmicas. Su eficacia está bien establecida, con una reducción de la sudoración del 82 al $87 \%$, aproximadamente, de 4 a 6 meses ${ }^{29,30}$. La dosis recomendada es de 50 a 75 UI en cada axila ${ }^{31}$. Los efectos secundarios son mínimos y el principal es el dolor ${ }^{32}$. Algunas intervenciones recomendadas para reducir la sensación de dolor durante el procedimiento son los anestésicos tópicos, el enfriamiento de la piel y la dilución de la toxina en lidocaína en lugar de solución salina.

\section{Tratamiento sistémico}

Agentes orales: los principales agentes sistémicos usados para tratar la hiperhidrosis focal son los anticolinérgicos, debido a que inhiben la señalización de acetilcolina a nivel neuroglandular. Entre los medicamentos orales disponibles para el tratamiento de la hiperhidrosis, se cuenta actualmente con agentes anticolinérgicos (oxibutinina, glicopirrolato, bromuro de mentatelina) y los agonistas alfa-adrenérgicos (cloni- dina). La principal limitación de estos medicamentos es la gran frecuencia de efectos secundarios, como boca seca, visión borrosa, retención urinaria, estreñimiento y taquicardia. Se prefiere su uso en la hiperhidrosis generalizada.

\section{Tratamiento quirúrgico}

Raspado (curettage) subcutáneo: consiste en el raspado (curettage) de las glándulas sudoríparas axilares $^{33}$. Es un procedimiento quirúrgico mínimamente invasivo, seguro, con baja frecuencia de efectos secundarios ${ }^{34}$ y de fácil realización. Cuando la técnica quirúrgica es correcta, tiene una efectividad del $100 \%$ para tratar de forma definitiva la hiperhidrosis axilar ${ }^{35,36}$.

Simpatectomía transtorácica: es la última opción terapéutica debido al riesgo de complicaciones posquirúrgicas, como hiperhidrosis compensatoria, sangrado, infección y neumotórax ${ }^{37}$. Es un tratamiento que ha tenido buen efecto en la hiperhidrosis palmar, así como en la axilar ${ }^{38}$.

\section{Termoterapia}

La amplia brecha existente entre el tratamiento conservador y el quirúrgico se ha cerrado en los últimos años con la termoterapia, es decir, el efecto terapéutico del calor sobre las glándulas sudoríparas. Esto ha sido técnicamente posible mediante el uso de láser ${ }^{39}$, microondas ${ }^{40}$ y radiofrecuencia ${ }^{41}$, gracias a que las proteínas son desnaturalizadas a temperaturas mayores de $56{ }^{\circ} \mathrm{C}$.

\section{Tratamiento láser}

La eficacia de los tratamientos con rayos láser para la hiperhidrosis axilar primaria aún es controversial. En varios estudios, se ha utilizado el láser Nd:YAG con parámetros similares a los utilizados para la depilación, con disminución subjetiva de la sudoración ${ }^{42}$. Sin embargo, la falta de estudios clínicos que permitan establecer un protocolo de tratamiento, especialmente en la población pediátrica, ha impedido que sea un tratamiento recomendado.

\section{Microondas}

Las microondas producen calor mediante la rotación física de moléculas dipolares, con una selectividad relativa por la dermis rica en agua y en las glándulas sudoríparas, y menor absorción en el tejido celular subcutáneo. Se ha propuesto en los últimos años un nuevo dispositivo que tiene la capacidad de destruir las glándulas ecrinas, con muy pocos efectos secundarios en el tejido adyacente ${ }^{43}$, que representa una opción terapéutica no invasiva y con menor incidencia de 
complicaciones.

En diferentes estudios clínicos se ha demostrado una eficacia cercana al $85 \%$ con solo cuatro sesiones, una a la semana ${ }^{44}$. Sin embargo, el seguimiento no ha superado los 12 meses, es decir, no conocemos por cuánto tiempo sea permanente el efecto y no se cuenta con estudios en la población colombiana.

\section{Radiofrecuencia}

El campo eléctrico oscila 40.000 veces por segundo. La oscilación de las moléculas de agua origina fricción, la cual, a su vez, genera calor efectivo en el tejido. La radiofrecuencia genera calor mediante dos mecanismos: el movimiento rotacional del agua en campos electromagnéticos (unipolar) y la resistencia tisular a la corriente conductiva (bipolar).

Mediante la termólisis, se compromete la actividad electroquímica de las glándulas sudoríparas. Hay estudios que demuestran cambios histopatológicos después de solo cuatro sesiones ${ }^{45}$. Dada su reciente aparición, existen pocos estudios que evalúen tanto su utilidad como los efectos secundarios ${ }^{46}$.

En la mayoría de estudios, se ha utilizado radiofrecuencia con microagujas (pixelada), la cual permite el contacto directo y un mejor control de la energía almacenada 47

En la población colombiana, no se cuenta con ningún estudio que evalúe la termoterapia con radiofrecuencia como tratamiento de la hiperhidrosis axilar primaria a largo plazo.

\section{CONCLUSIONES}

La hiperhidrosis axilar primaria puede alterar la calidad de vida de los pacientes.

Existen varios tratamientos disponibles para la hiperhidrosis axilar primaria, con efectos temporales, lo que ha motivado el desarrollo de nuevas técnicas que ofrezcan mejores resultados.

El tratamiento tópico es fácil de aplicar y de relativo bajo costo. Se considera la primera línea de tratamiento para la hiperhidrosis axilar primaria. El cloruro de aluminio es el agente más frecuentemente utilizado, pero solo sirve para casos leves. Surge ahora el glicopirrolato en atomizaciones, con buena reacción terapéutica.

La eficacia de la toxina botulínica está bien establecida. Es un procedimiento de alto costo y su efecto dura de cuatro a seis meses, aproximadamente. Los efectos secundarios en las axilas son mínimos.

La termoterapia (láser, microondas, radiofrecuencia pixelada) se ha utilizado enlos últimos años con re- sultados muy variables y, aunque es claro su efecto en cuanto a destrucción de las glándulas, aún no se dispone de suficientes datos sobre la duración del efecto, la temporalidad de las sesiones, y la necesidad y frecuencia del mantenimiento.

Es muy importante hacer estudios en nuestra población. El tratamiento debe ser individualizado y se debe informar al paciente sobre la cronicidad de la enfermedad y todas las opciones terapéuticas de las que se dispone, con sus limitaciones y efectos secundarios.

\section{REFERENCIAS}

1. Leong L. Human thermoregulation and measurement of body temperature in exercise and clinical settings. Ann Acad Med Singapore. 2008;37:347-53.

2. Atkins JL, Butler PE. Hyperhidrosis: A review of current management. Plast Reconstr Surg. 2002;110:222-8.

3. Solish N, Bertucci V, Dansereau A, Hong HC, Lynde $\mathrm{C}$, et al. A comprehensive approach to the recognition and severity-based treatment of focal hyperhidrosis: Recommendations of the Canadian Hyperhidrosis Advisory Committee. Dermatol Surg. 2007;33:908-23.

4. Mahendiran S, Burkhart CN,. Burkhart CG. Hyperhidrosis: A review of a medical condition. The Open Dermatology Journal. 2009;3:195-7.

5. Strutton DR, Kowalski J, Glasser DA, Stang P. US prevalence of hyperhidrosis and impact on individuals with axillary hyperhidrosis: Results from a national survey. J Am Acad Dermatol. 2004;51:241-8.

6. Janig W. Functions of the sympathetic innervation of the skin. In: Loewy $\mathrm{AD}$, editor. Central regulation of autonomic functions. New York: Oxford University Press; 1990. p. 334-48.

7. Simon E. Temperature regulation: The spinal cord as a site of extrahypothalamic thermoregulatory functions. Rev Physiol Biochem Pharmacol. 1974;71:1-76.

8. Moran DS, Mendal L. Core temperature measurement: Methods and current insights. Sports Med. 2002;32:879-85.

9. Schnider P, Binder M, Auff E, Kittler H, Berger T, Wolff K. Double-blind trial of botulinum, a toxin for the treatment of focal hyperhidrosis of the palms. Br J Dermatol. 1997;136:548-52.

10. Fawcett DW. Tratado de histología. $12^{\mathrm{a}}$ edición. Madrid: Editorial McGraw Hill Interamericana; 
1995. p. $703-8$.

11. Altman RS, Schwartz RA. Emotionally induced hyperhidrosis. Cutis. 2002;69:336-8.

12. Felini R, Demarchi AR, Fistarol ED, Matiello M, Delorenze LM. Prevalence of hyperhidrosis in the adult population of Blumenau, SC, Brazil. An Bras Dermatol. 2009;84:361-6.

13. Sato K, Kang WH, Saga K, Sato KT. Biology of sweat glands and their disorders. II. Disorders of sweat gland function. J Am Acad Dermatol. 1989;20;713-26.

14. Walling HW. Primary hyperhidrosis increases the risk of cutaneous infection: A case-control study of 387 patients. J Am Acad Dermatol. 2009;61:242-6.

15. Walling HW. Clinical differentiation of primary from secondary hyperhidrosis. J Am Acad Dermatol. 2011;64:690-5.

16. Tugnoli V, Eleopra R, De Grandis D. Hyperhidrosis and sympathetic skin response in chronic alcoholic patients. Clin Auton Res. 1999;9:17-22.

17. Hornberger J, Grimes K, Naumann M, Glaser DA, Lowe NJ, Naver H, et al. Recognition, diagnosis, and treatment of primary focal hyperhidrosis. J Am Acad Dermatol. 2004;51;274-86.

18. Lewis V, Finlay AY. 10 years' experience of the Dermatology Life Quality Index (DLQI). J Investig Dermatol Symp Proc. 2004;9:169-80.

19. de Campos JRM, Kauffman P, de Campos WE, Andrade FLO, Kuzniek S, Wolosker N, et al. Questionnaire of quality of life in patients with primary hyperhidrosis. J Pneumologia. 2003;29:178-81.

20. Swartling C, Naver H, Lindberg M. Botulinum A toxin improves life quality in severe primary focal hyperhidrosis. Eur J Neurol. 2001;8:247-52.

21. Finlay AY, Khan GK. Dermatology Life Quality Index (DLQI): A simple practical measure for routine clinical use. Clin Exp Dermatol. 1994;19:210-6.

22. Vorkamp T, Foo FJ, Khan S, Schmitto JD, Wilson P. Hyperhidrosis: Evolving concepts and a comprehensive review. Surgeon. 2010;8:287-92.

23. Shelley WB, Hurley HJ, Jr. Studies on topical antiperspirant control of axillary hyperhidrosis. Acta Derm Venereol. 1975;55:241-6o.

24. Baker DM. Topical glycopyrrolate reduces axillary hyperhidrosis. J Eur Acad Dermatol Venereol. 2016;30:2131-6.

25. Stolman LP. Hyperhidrosis: Medical and surgical treatment. Eplasty. 2008;18:e22

26. Reinauer S, Neusser A, Schauf G, Holzle E. Ion- tophoresis with alternating current and direct current offset (AC/DC iontophoresis): A new approach for the treatment of hyperhidrosis. $\mathrm{Br} \mathrm{J}$ Dermatol. 1993;129:166-9.

27. Karakoc Y, Aydemir EH, Kalkan MT, Unal G - Safe control of palmo-plantar hyperhidrosis with direct electrical current. Int J Dermatol. 2002;41:602-5.

28. Simpson LL. The origin, structure, and pharmacological activity of botulinum toxin. Pharmacol Rev. 1981;33:155-8.

29. Heckmann M, Ceballos-Baumann AO, Plewig G. Botulinum toxin A for axillary hyperhidrosis (excessive sweating). N Engl J Med. 2001;322:48893.

30. Naver H, Swartling C, Aquilonius SM. Palmar and axillary hyperhidrosis treated with botulinum toxin: one-year clinical follow-up. Eur J Neurol 2000;7:55-62.

31. Naumann M, Lowe NJ. Botulinum toxin type A in treatment of bilateral primary axillary hyperhidrosis: Randomised, parallel group, double blind, placebo controlled trial. Br Med J. 2001;323:596.

32. Naumann M, Lowe NJ, Kumar CR, Hamm H. Botulinum toxin type $\mathrm{A}$ is a safe and effective treatment for axillary hyperhidrosis over 16 months: A prospective study. Arch Dermatol. 2003;139:731-6.

33. Jemec B, Holm Hansen B. Follow-up of patients operated on for axillary hyperhidrosis by subcutaneous curettage. Scand J Plast Reconstr Surg. 1978;12:65-7.

34. Darabaneanu S, Darabaneanu HA, Niederberger U, Russo PA, Lischner S, Hauschild A. Long-term efficacy of subcutaneous sweat gland suction curettage for axillary hyperhidrosis: A prospective gravimetrically controlled study. Dermatol Surg. 2008;34:1170-7.

35. Böni R. Tumescent suction curettage in the treatment of axillary hyperidrosis: Experience in 63 patients. Dermatology. 2006;213:215-7.

36. Hernández C, Gómez L, Londoño Á. Evaluación de la técnica quirúrgica "curetaje" axilar para el tratamiento de la hiperhidrosis axilar, fase retrospectiva. Rev Asoc Colomb Dermatol. 2012;20:314-21.

37. Baram A. Single incision thoracoscopic sympathectomy for palmar and axillary hyperhidrosis. SAGE Open Medicine. 2014;2:2050312114523757.

38. Licht, PB. Thoracoscopic sympathectomy for 
axillary hyperhidrosis: The influence of T4. Ann Thorac Surg. 2005;80:455-60.

39. Goldman A, Wollina U. Subdermal Nd-YAG laser for axillary hyperhidrosis. Dermatol Surg. 2008;34:756-62.

40. Lupin M, Hong HC, O’Shaughnessy KF. Longterm efficacy and quality of life assessment for treatment of axillary hyperhidrosis with a microwave device. Dermatol Surg. 2014;40:805-7.

41. Fatemi-Naeini F, Abtahi-Naeini B, Pourazizi M, Nilforoushzadeh, Mirmohammdkhani M. Fractionated microneedle radiofrequency for treatment of primary axillary hyperhidrosis: A sham control study. Australas J Dermatol. 2015;56:279-84.

42. Letada PR, Landers JT, Uebelhoer NS, Shumaker PR. Treatment of focal axillary hyperhidrosis using a long pulsed Nd:YAG 1,064 nm laser at hair reduction settings. J Drugs Dermatol. 2012;11:59-63.

43. Johnson JE, O'Shaughnessy KF, Kim S. Microwave thermolysis of sweat glands. Lasers Surg Med. 2012;44:20-5.

44. Glaser DA, Coleman WP, Fan LK, Kaminer MS, Kilmer SL, Nossa R, Smith SR, O’Shaughnessy $\mathrm{KF}$. A randomized, blinded clinical valuation of a novel microwave device for treating axillary hyperhidrosis: The dermatologic reduction in underarm perspiration study. Dermatol Surg. 2012;38:185-91.

45. Naeini FF, Saffaei A, Pourazizi M, Abtahi-Naeini B. Histopathological evidence of efficacy of microneedle radiofrequency for treatment of axillary hyperhidrosis. Indian J Dermatol Venereol Leprol. 2015;81:288-90.

46. Pinson I, Olisova O. Non-invasive short-wave guided radiofrequency device for long-term sweat reduction in patients with primary axillary hyperhidrosis: A preliminary study. Moscow: Alma Laser Ltd.; 2013.

47. Kim M, Shin JY. Efficacy of fractional microneedle. Radiofrequency device in the treatment of primary axillary hyperhidrosis: A pilot study. Dermatology. 2013;227:243-9. 